\title{
Recent Advances in Cross-linked Polyethylene Based Nanocomposites for High Voltage Engineering Applications: A Critical Review
}

Jince Thomas ${ }^{1,5}$, Blessy Joseph ${ }^{1}$, Josmin. P. Jose ${ }^{2}$, Hanna J Maria ${ }^{1}$, Priyanka Main ${ }^{3}$, Azerai Ali Rahman ${ }^{4}$, Bejoy Francis ${ }^{5}$, Zakiah Ahmad ${ }^{*}$, Sabu Thomas ${ }^{1,7,8 *}$

1. International and Inter University Center for Nanoscience and Nanotechnology, Mahatma Gandhi University, Kottayam, Kerala, India.

2. Mar Thoma College, Thiruvalla, Kerala, India.

3. Hindustan Aeronautics Limited (HAL), Bengaluru, India

4. Faculty of Civil Engineering, Universiti Teknologi Mara, Shah Alam, Selangor, Malaysia.

5. Department of Chemistry, St. Berchmans College, Changanassery, Kerala, India.

6. Institute of Infrastructure Engineering and sustainable Management, Universiti Teknologi Mara, Shah Alam, Selangor, Malaysia.

7. School of Chemical Sciences, Mahatma Gandhi University, Kottayam, Kerala, India.

8. School of Energy Materials, Mahatma Gandhi University, Kottayam, Kerala, India. 


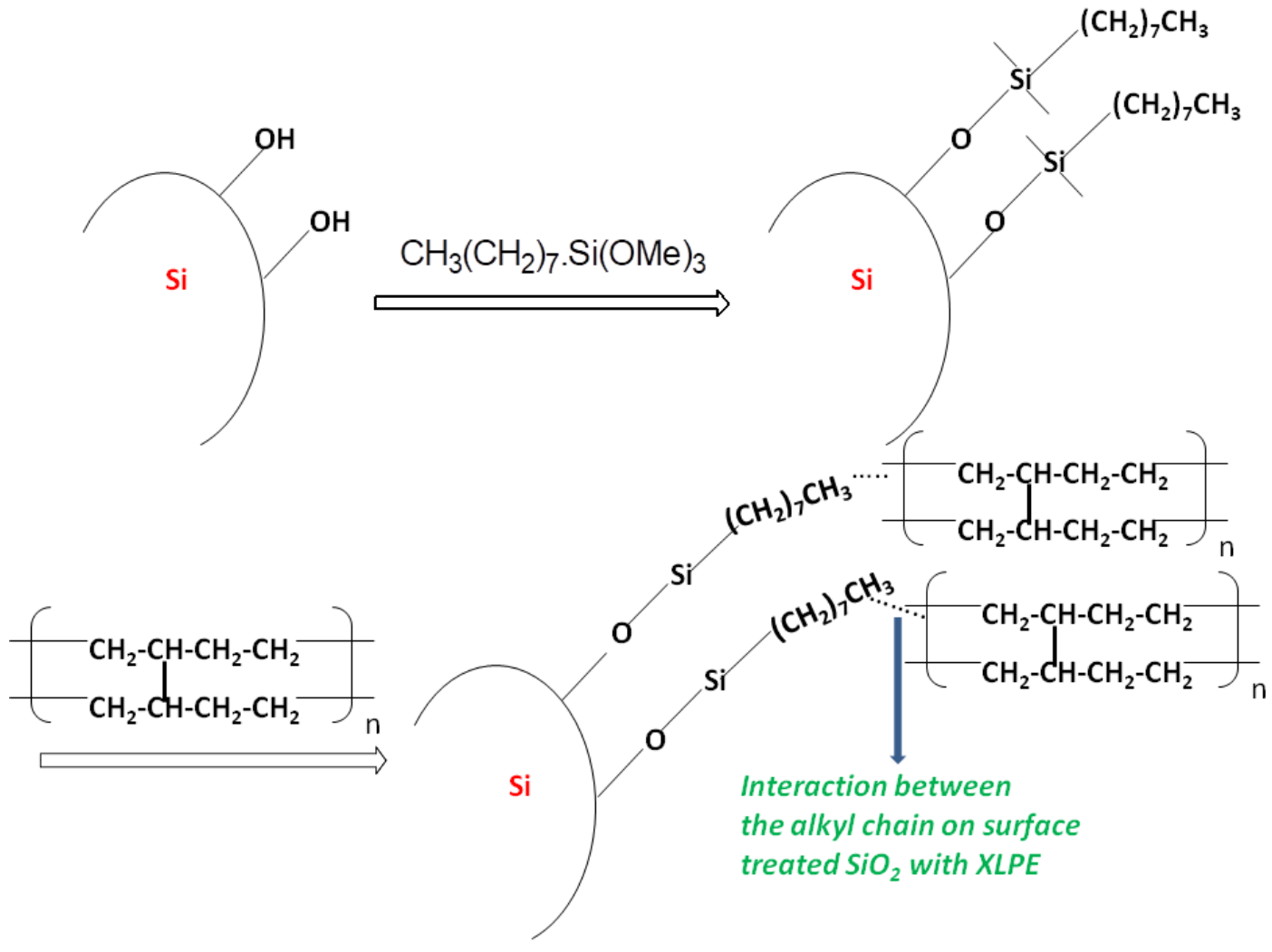

Figure S1: Interaction between surface modified $\mathrm{SiO}_{2}$ and XLPE chain( Adapted with permission from ref. ${ }^{1}$ Copyright 2014 , The Royal Society of Chemistry. ) 
Table S1. Advantages and disadvantages of materials used in high voltage cable insulation fields.

\begin{tabular}{|c|c|c|c|}
\hline Materials used & Advantages & Limitations & Reference \\
\hline $\begin{array}{c}\text { Paper-Insulated } \\
\text { Lead-Covered cables } \\
\text { (PILC) }\end{array}$ & $\begin{array}{l}\text { Long-term reliability } \\
\text { and their ability to fit } \\
\text { it into older conduit- } \\
\text { manhole systems. }\end{array}$ & $\begin{array}{c}\text { Corrosion of the lead } \\
\text { sheath on } \\
\text { PILC cables. }\end{array}$ & 2,3 \\
\hline $\begin{array}{l}\text { Polyvinyl chloride } \\
\text { (PVC) }\end{array}$ & $\begin{array}{c}\text { Cheap, Durable, widely } \\
\text { available }\end{array}$ & $\begin{array}{l}\text { Highest dielectric } \\
\text { losses, melts at high } \\
\text { temperatures, } \\
\text { contains halogens. }\end{array}$ & 4,5 \\
\hline Polyethylene (PE) & $\begin{array}{l}\text { Lowest dielectric } \\
\text { losses, high initial } \\
\text { dielectric strength }\end{array}$ & $\begin{array}{l}\text { Highly sensitive to } \\
\text { water treeing, } \\
\text { material breaks down } \\
\text { at high temperatures }\end{array}$ & 6,7 \\
\hline $\begin{array}{c}\text { Ethylene- Propylene } \\
\text { Rubber (EPR) }\end{array}$ & $\begin{array}{l}\text { Increased flexibility, } \\
\text { low sensitivity to } \\
\text { water treeing }\end{array}$ & $\begin{array}{l}\text { Medium-high } \\
\text { dielectric losses }\end{array}$ & 8,9 \\
\hline $\begin{array}{c}\text { Crosslinked } \\
\text { Polyethylene (XLPE) }\end{array}$ & $\begin{array}{l}\text { Higher operational } \\
\text { temperature, } \\
\text { mechanically robust } \\
\text { and weightless, }\end{array}$ & $\begin{array}{c}\text { Space charge } \\
\text { accumulation and } \\
\text { stress inversion } \\
\text { phenomena }\end{array}$ & 10,11 \\
\hline
\end{tabular}




\begin{tabular}{|l|l|l|}
\hline & $\begin{array}{c}\text { installation process is } \\
\text { simpler and faster }\end{array}$ & \\
\hline
\end{tabular}

Table S2. A brief insulation property comparison of XLPE with other insulation materials

\begin{tabular}{|c|c|c|c|c|c|}
\hline \multirow{2}{*}{ Properties } & \multicolumn{5}{|c|}{ Insulation Materials } \\
\cline { 2 - 6 } & PVC & EPR & $\begin{array}{c}\text { Impregnated } \\
\text { paper }\end{array}$ & LDPE & XLPE \\
\hline Permittivity & 8 & 3.3 & 3.5 & 2.3 & 4 \\
\hline $\begin{array}{c}\text { Dielectric } \\
\text { loss tangent }\end{array}$ & 1000 & 40 & 20 & 4 & \\
\hline $\begin{array}{c}\left(10^{-4}\right) \\
\text { Thermal }\end{array}$ & 6 & 5 & $5-6$ & 3 & 3.5 \\
\hline $\begin{array}{c}\text { resistivity } \\
\left({ }^{\circ} \mathrm{CmW}^{-1}\right)\end{array}$ & & & & & \\
\hline
\end{tabular}


Table S3. The failure mechanism and their effects in cable insulation systems

\begin{tabular}{|c|c|c|}
\hline Failure factor & Mechanism & Effects \\
\hline \multicolumn{3}{|c|}{ Thermal } \\
\hline High temperature & $\begin{array}{l}\text { Chemical reaction, } \\
\text { incompatibility of materials, } \\
\text { thermal expansion (radial and } \\
\text { axial), diffusion. }\end{array}$ & $\begin{array}{l}\text { Hardening, softening, loss of } \\
\text { mechanical strength, } \\
\text { embrittlement, increased tan } \\
\text { delta, shrinkage, loss of } \\
\text { adhesion, separation, } \\
\text { delamination at interfaces, } \\
\text { conductor penetration, } \\
\text { formation of soft spots, } \\
\text { wrinkles. }\end{array}$ \\
\hline Low temperature & Cracking, thermal contraction. & $\begin{array}{l}\text { Shrinkage, loss of adhesion, } \\
\text { separation, delamination at } \\
\text { interfaces. }\end{array}$ \\
\hline \multicolumn{3}{|c|}{ Electrical } \\
\hline $\begin{array}{l}\text { Voltage, AC, DC, } \\
\text { impulse }\end{array}$ & $\begin{array}{c}\text { Partial discharges } \\
(\mathrm{PD}), \text { electrical treeing }(\mathrm{ET}) \\
\text { water treeing (WT), dielectric } \\
\text { losses and capacitance charge } \\
\text { injection ,intrinsic breakdown }\end{array}$ & $\begin{array}{l}\text { PD and ET increased ,thermal } \\
\text { ageing, immediate failure }\end{array}$ \\
\hline \multicolumn{3}{|c|}{ Mechanical } \\
\hline $\begin{array}{c}\text { Tensile, compressive, shear } \\
\text { stresses, Fatigue, cyclic }\end{array}$ & Cracking, rupture & $\begin{array}{l}\text { Mechanical rupture, loss of } \\
\text { adhesion, separation, }\end{array}$ \\
\hline
\end{tabular}




\begin{tabular}{|c|c|c|}
\hline bending, vibration & & delamination at interfaces \\
\hline \multicolumn{3}{|c|}{ Environmental } \\
\hline $\begin{array}{l}\text { Water/humidity } \\
\text { Liquids/gases } \\
\text { contamination }\end{array}$ & $\begin{array}{l}\text { Dielectric losses and } \\
\text { capacitance, electrical } \\
\text { tracking, water treeing, } \\
\text { corrosion }\end{array}$ & $\begin{array}{c}\text { Increased temperature, } \\
\text { thermal ageing, thermal } \\
\text { runaway, increased losses and } \\
\text { ET }\end{array}$ \\
\hline
\end{tabular}

Table S 4. Changes in electrical properties of XLPE nanocomposites

\begin{tabular}{|c|c|c|}
\hline Property & Outcome & $\begin{array}{c}\text { Reason for change in } \\
\text { properties }\end{array}$ \\
\hline Permittivity & Increase/Decrease & $\begin{array}{c}\text { Different permittivity values } \\
\text { and weight percentage of } \\
\text { nanoparticles }\end{array}$ \\
\hline Dielectric loss & $\begin{array}{c}\text { Well within the range of } \\
\text { Insulators }\end{array}$ & $\begin{array}{c}\text { Interface structures between } \\
\text { XLPE and nanofiller }\end{array}$ \\
\hline Partial discharge resistance & Increase & $\begin{array}{l}\text { Nano segmentation of XLPE, } \\
\text { shape of the nanofiller and } \\
\text { electric field at interfacial } \\
\text { region }\end{array}$ \\
\hline Treeing resistance & Increase & $\begin{array}{l}\text { Nano segmentation of XLPE, } \\
\text { shape of the nanofiller and } \\
\text { electric field at interfacial } \\
\text { region }\end{array}$ \\
\hline Breakdown strength & Increase or no change & $\begin{array}{l}\text { Coupling, impurity effects } \\
\text { and loading weight } \\
\text { percentage of nanoparticles }\end{array}$ \\
\hline DC conductivity & Increase or no change & $\begin{array}{c}\text { Roles of ion traps and } \\
\text { impurities }\end{array}$ \\
\hline
\end{tabular}


Table S 5. Electrical, mechanical and thermal properties of XLPE nanocomposites

\begin{tabular}{|c|c|c|c|}
\hline \multirow{2}{*}{$\begin{array}{c}\text { PE based } \\
\text { nanocomposites }\end{array}$} & \multicolumn{3}{|c|}{ Properties } \\
\hline & $\begin{array}{c}\text { Electrical (DC } \\
\text { breakdown } \\
\text { strength) } \mathrm{kV} / \mathrm{mm}\end{array}$ & $\begin{array}{c}\text { Mechanical } \\
\text { (Tensile } \\
\text { strength) } \mathrm{MPa}\end{array}$ & $\begin{array}{c}\text { Thermal } \\
\text { stability }\left({ }^{\circ} \mathrm{C}\right)\end{array}$ \\
\hline $\begin{array}{c}\mathrm{XLPE}+\mathrm{SiO}_{2} \\
\text { (surface modified) }\end{array}$ & $\begin{array}{c}446\left(25^{\circ} \mathrm{C}\right), 422 \\
\left(60^{\circ} \mathrm{C}\right), 220\left(80^{\circ} \mathrm{C}\right)^{12}\end{array}$ & $10.3^{13}$ & $\begin{array}{c}\text { Stable up to } \\
540^{13}\end{array}$ \\
\hline XLPE+ MgO & $57^{14}$ & $12^{15}$ & $550^{15}$ \\
\hline $\mathrm{LDPE}+\mathrm{MgO}$ & $\begin{array}{c}28 \text { (AC breakdown } \\
\text { strength })^{14}\end{array}$ & $12^{16}$ & $520^{16}$ \\
\hline $\mathrm{LDPE}+\mathrm{SiO}_{2}$ & $\begin{array}{c}\text { 7(AC breakdown } \\
\text { strength })^{14}\end{array}$ & $10.79^{17}$ & $492^{18}$ \\
\hline $\begin{array}{c}\text { LDPE +MMT (5 } \\
\text { wt } \%)\end{array}$ & $\begin{array}{c}\text { 19(AC breakdown } \\
\text { strength) }{ }^{14}\end{array}$ & $13.6^{19}$ & $480^{19,20}$ \\
\hline $\begin{array}{c}\text { PE+MMT (20 } \\
\text { wt } \%)\end{array}$ & $\begin{array}{c}20 \text { (AC breakdown } \\
\text { strength })^{14}\end{array}$ & $\begin{array}{l}18.8 \text { (Yield } \\
\text { strength) }{ }^{21}\end{array}$ & $455^{21}$ \\
\hline
\end{tabular}


Table S 6. Lists of nanoparticles used in XLPE system

\begin{tabular}{|c|c|c|}
\hline Nanoparticles & Findings & Reference \\
\hline $\mathrm{CaCO}_{3}$ & $\begin{array}{l}\text { Improvement in the } \\
\text { electrical } \\
\text { performance of cross- } \\
\text { linked polyethylene } \\
\text { high voltage cables. }\end{array}$ & 22,23 \\
\hline Carbon black & $\begin{array}{l}\text { Decrease of dielectric } \\
\text { constant with } \\
\text { frequency. }\end{array}$ & 24 \\
\hline Graphene Oxide & $\begin{array}{l}\text { Improvements in } \\
\text { thermal stability and } \\
\text { fire safety properties } \\
\text { in nonpolar polymers. }\end{array}$ & 25,26 \\
\hline $\mathrm{ZnO}$ & $\begin{array}{c}\text { Enhanced DC } \\
\text { dielectric breakdown } \\
\text { strength. }\end{array}$ & 27,28 \\
\hline $\mathrm{TiO}_{2}$ & $\begin{array}{c}\text { Space charge } \\
\text { accumulation was } \\
\text { suppressed by filling } \\
\mathrm{TiO}_{2} \text { nanoparticles }\end{array}$ & 29 \\
\hline
\end{tabular}


Table S 7. Young's modulus of XLPE (X), XLPE/Al ${ }_{2} \mathrm{O}_{3}$ (A), XLPE/clay (C), XLPE/Al ${ }_{2} \mathrm{O}_{3}$ : clay $=1: 1(\mathrm{~A} 1 \mathrm{C}), \mathrm{XLPE} / \mathrm{Al}_{2} \mathrm{O}_{3}:$ clay $=2: 1$ (A2C) $\left(\right.$ Reprinted with permission from ref. ${ }^{1}$ .Copyright 2014, The Royal Society of Chemistry.)

\begin{tabular}{|c|c|}
\hline Sample & Young's modulus (MPa) \\
\hline $\mathrm{X}$ & $263 \pm 10$ \\
\hline C & $370 \pm 8$ \\
\hline A & $384 \pm 6$ \\
\hline A1C & $812 \pm 9$ \\
\hline A2C & $555 \pm 11$ \\
\hline
\end{tabular}




\section{References}

(1) Jose, J. P.; Thomas, S., Alumina-clay Nanoscale Hybrid Filler Assembling in CrossLinked Polyethylene Based Nanocomposites: Mechanics and Thermal Properties. Phys. Chem. Chem. Phys.2014, 16, 14730.

(2) Dyba, J., The Rise and Decline in The United States of Impregnated Paper Insulated Metallic Sheathed Cable, Solid-type. I. IEEE Electr.Insul. Mag. 1999, 15, 13.

(3) Goodwin, F.; Dyba, J. In Recent Technological Developments for Lead Sheathed Cables, Proceedings of IEEE/PES Transmission and Distribution Conference, IEEE; 1994, 568-573.

(4) Beneš, M.; Plaek, V.; Matuschek, G.; Kettrup, A.; Györyová, K.; Emmerich, W.; Balek, V. Lifetime Simulation and Thermal Characterization of PVC Cable Insulation Materials. $J$. Therm. Anal.Calorim. 2005, 82, 761.

(5) Yaacob, M.; Sin, L.; Aman, A. In A New Polyvinyl Chloride Cable Insulation Using Micro and Nano Filler Materials, 4th International Power Engineering and Optimization Conference (PEOCO), IEEE; 2010,221-225.

(6) Vahlstrom, W., Investigation of Insulation Deterioration in $15 \mathrm{kV}$ and $22 \mathrm{kV}$ Polyethylene Cables Removed from Service. IEEE Trans.Power Appar. Sys. 1972, 3, 1023.

(7) Serra, S.; Montanari, G.; Mazzanti, G. Theory of Inception Mechanism and Growth of Defect-induced Damage in Polyethylene Cable Insulation. J.Appli.Phys.2005, 98, 034102.

(8) Brown, M. Performance of Ethylene-propylene Rubber Insulation in Medium and High Voltage Power Cable. IEEE Trans.Power Appar. Sys. 1983, 2, 373.

(9) Mizuno, Y.; Nagao, M.; Kosaki, M.; Shimizu, N.; Horii, K. Evaluation of EthylenePropylene Rubber as Electrical Insulating Material for A Superconducting Cable. IEEE Trans. Electr. Insul.1992, 27, 1108.

(10) Chen, G.; Hao, M.; Xu, Z.; Vaughan, A.; Cao, J.; Wang, H. Review of High Voltage Direct Current Cables.CSEE J. Power Energy Sys. 2015, 1, 9. 
(11) Fabiani, D.; Montanari, G. C.; Laurent, C.; Teyssedre, G.; Morshuis, P.; Bodega, R.; Dissado, L.; Campus, A.; Nilsson, U. Polymeric HVDC Cable Design and Space Charge Accumulation. Part 1: Insulation/Semicon Interface. IEEE Electr.Insul. Mag.2007, 23, 11.

(12) Roy, M.; Nelson, J. K.; MacCrone, R.; Schadler, L. Candidate Mechanisms Controlling the Electrical Characteristics of Silica/XLPE Nanodielectrics. J. Mater.Sci.2007, 42,3789.

(13) Jose, J. P.; Ahmad, Z.; Thomas, S., Hybrid Nanoparticle-Based XLPE/SiO2/TiO2 and XLPE/SiO2 Nanocomposites: Nanoscale Hybrid Assembling, Mechanics and Thermal properties. In InCIEC Springer; 2014, 895-902.

(14) Paramane, A. S.; Kumar, K. S., A review on Nanocomposite Based Electrical Insulations. Trans. Electr. Electron. Mater.2016, 17, 239.

(15) Peng, S.; He, J.; Hu, J.; Huang, X.; Jiang, P. Influence of Functionalized MgO Nanoparticles on Electrical Properties of Polyethylene Nanocomposites. IEEE Trans. Dielec. Electr. Insul. 2015, 22, 1512.

(16) Reddy, C. C.; Gosyowaki, M.; Murata, Y.; Sekiguchi, Y. In Superior Thermal Breakdown Performance of MgO-LDPE Nanocomposite Materials for HVDC Insulation.International Symposium on Electrical Insulating Materials (ISEIM 2008), IEEE; 2008, 661-664.

(17) Redhwi, H. H.; Siddiqui, M. N.; Andrady, A. L.; Hussain, S. Durability of LDPE Nanocomposites with Clay, Silica, and Zincoxide: Part I: Mechanical Properties of the Nanocomposite Materials. J. Nanomaterials. 2013, 21.

(18) Wang, Y.; Wang, C.; Zhang, Z.; Xiao, K. Effect of Nanoparticles on the Morphology, Thermal, and Electrical Properties of Low-density Polyethylene After Thermal Aging. Nanomaterials, 2017, 7, 320.

(19) Monica A, P.; Bernabé L, R.; Karla A, G.-M.; Víctor H, C.-R.; Miguel, M.; Johanna, C.; Álvaro, M., Low Density Polyethylene (LDPE) Nanocomposites with Passive and Active Barrier Properties. J.Chile.Chem.Soc. 2014, 59, 2442.

(20) Liu, S.-P. Flame Retardant and Mechanical Properties of Polyethylene/Magnesium hydroxide/Montmorillonite Nanocomposites. J. Ind.Eng.Chem. 2014, 20, 2401.

(21) Araújo, E.; Barbosa, R.; Rodrigues, A.; Melo, T.; Ito, E. Processing and Characterization of Polyethylene/Brazilian Clay Nanocomposites. Materials Science and Engineering: A. 2007, 445,141 . 
(22) Habib, M.; Nasart, L.; Sharkawy, R. In Improvement The Electrical Performance of Cross-linked Polyethylene High Voltage Cables, Power Systems Conference (MEPCON), Eighteenth International Middle East, IEEE; 2016; 21-25.

(23) Ding, J.; Ma, W.; Song, F.; Zhong, Q. Effect of Nano-calcium Carbonate on Microcellular Foaming of Polypropylene. J.Mater.Sci.2013, 48, 2504.

(24) Moon, K. S.; Do Choi, H.; Jung, H. Y.; Cho, K. Y.; Yoon, H. G.; Moon, T. J. Dielectric Properties of Human Phantom Material Using XLPE Composite With Shape Memory Characteristics. Polymer (Korea).1999, 23, 763.

(25) Hu, W.; Zhan, J.; Wang, X.; Hong, N.; Wang, B.; Song, L.; Stec, A. A.; Hull, T. R.; Wang, J.; Hu, Y. Effect of Functionalized Grapheneoxide With Hyper-branched Flame Retardant on Flammability and Thermal Stability of Cross-linked Polyethylene. Ind.Eng.Chem. Res.2014, $53,3073$.

(26) Tripathi, S. N.; Saini, P.; Gupta, D.; Choudhary, V. Electrical and Mechanical Properties of PMMA/reduced Graphene oxide Nanocomposites Prepared via in situ Polymerization. J. Mater Sci. 2013, 48, 6223-6232.

(27) Kim, Y.-M.; Cha, Y.-K.; Lim, K.-J.; Nam, J.-H.; Lee, G.-J. In Electrical Insulation Evaluation of Crosslinked Polyethylene Nanocomposite Blended with ZnO, International Conference on Condition Monitoring and Diagnosis (CMD), IEEE; 2012, 1242-1245.

(28) Hong, J.; Schadler, L.; Siegel, R.; Mårtensson, E. Electrical Behavior of Low Density Polyethylene Containing an Inhomogeneous Distribution of $\mathrm{ZnO}$ nanoparticles. J.Mater. Sci. 2006, 41,5810 .

(29) Wang, Y.; Xiao, K.; Wang, C.; Yang, L.; Wang, F. Effect of Nanoparticle Surface Modification and Filling Concentration on Space Charge Characteristics in TiO2/XLPE nanocomposites. J. Nanomaterials. 2016. 